\title{
ОЦЕНКА СТЕПЕНИ ФУНКЦИОНАЛЬНОЙ НЕСТАБИЛЬНОСТИ ПРИ ОБОСТРЕНИИ ИБС
}

\author{
(С Сараев И.А. ${ }^{1}$ Вишневский В.И. ${ }^{2}$
}

\author{
${ }^{1}$ Кафедра внутренних болезней № 2 Курского государственного медицинского университета, Курск; \\ ${ }^{2}$ кафедра внутренних болезней Орловского государственного университета им. И.С. Тургенева, Орел \\ E-mail: igorsarayev@yandex.ru
}

В статье приводятся данные о возможности диагностики глубины функциональных расстройств у 60 больных ИБС на фоне развития острого коронарного синдрома. Показано, что анализ нелинейной динамики информационного показателя кардиоритма - энтропии служит основой для представления системного гомеокинеза в виде аттракторной модели, обладающей измеряемыми топологическими признаками. Установлено, что развитие не-Q инфаркта миокарда как исхода острого коронарного синдрома без подъема сегмента ST к концу вторых суток сопровождается достоверно большей функциональной неустойчивостью по сравнению с таковой у лиц без необратимых поражений миокарда, несмотря наличие у них затянувшегося ангинального синдрома, что соответствует статистически значимым изменениям топологии аттрактора динамики энтропии кардиоритма. Предложенные количественные показатели, отражающие данное явление, могут рассматриваться как дополнительные альтернативные информативные маркеры как самого эффекта неустойчивости организации системного гомеостатического регулирования на фоне острой соматической патологии, так и служить ее количественным мерилом.

Ключевые слова: острый коронарный синдром, не-Q инфаркт миокарда, нестабильная стенокардия, количественная оценка функциональных расстройств, случайно-подобная динамика системного гомеокинеза, аттрактор энтропии синусового кардиоритма.

\section{EVALUATION OF THE FUNCTIONAL INSTABILITY DEGREE IN EXACERBATION OF CHD

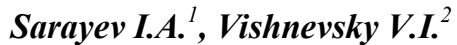 \\ ${ }^{1}$ Department of Internal diseases $\mathbf{N}$ of Kursk State Medical University, Kursk; \\ ${ }^{2}$ Department of Internal Diseases of Orel State University named after I.S. Turgenev, Orel}

The article presents data on the possibility of diagnosing the depth of functional disorders revealed in 60 patients with coronary artery disease against the background of developing acute coronary syndrome. It is shown that the analysis of the nonlinear dynamics of the information indicator of cardiorhythm - entropy serves as a basis for the presentation of systemic homeokinesis in the form of its attractor model with measurable topological features. It was established that the development of non-Q myocardial infarction as an outcome of acute coronary syndrome without ST segment elevation by the end of the second day is accompanied by significantly greater functional instability in comparison with that in persons without irreversible myocardial lesions, which corresponds to statistically significant changes in the topology of the attractor of entropy dynamics of cardiorhythm. The proposed quantitative indicators reflecting this phenomenon can be considered as additional alternative informative markers as the very effect of instability of the organization of systemic homeostatic regulation against the background of acute somatic pathology, and can serve as its quantitative measure.

Keywords: acute coronary syndrome, non-Q myocardial infarction, unstable angina, quantitative evaluation of functional disorders, random-like dynamics of systemic homeokinesis, attractor of entropy of sinus cardiorhythm.

Принципиальным условием эффективности мер по контролю объективного статуса больных с опасным в отношении летальности обострением соматических заболеваний, в частности - артериальной гипертонии, ИБС, является реальное представление о глубине функциональных расстройств, вызванных этими состояниями [1]. Синдромы, нередко требующие ургентного вмешательства и развивающиеся у конкретных больных, в рамках нозологических форм коронарной болезни индивидуально разнообразны. Тем не менее, в основе внезапного появления аритмий, острой сердечной недостаточности, рецидивирующих острых коагуляционных эпизодов и других клинических феноменов лежит общий механизм, представляющий собой упреждающее любую соматическую катастрофу расстройство системного гомеостатического регулирования. Адекватной моделью для анализа этого явления может служить такая, как правило, внезапно возникающая и динамическая форма ИБС как острый коронарный синдром (ОКС). Результат этого варианта острой коронарной патологии наиболее неоднозначен при отсутствии яркого маркера ишемии миокарда - подъема сегмента ST $[2,3]$. Нередко в основе случаев острого коронарного синдрома без подъема сегмента ST (OKC БПST) лежит острая неполная окклюзия одной из ветвей коронарного русла, а симптоматика заболевания может развиваться двояко: как проявление не-Q инфаркта миокарда (не-Q ИМ) или нестабильной стенокардии (НС). Усугубление коронарной патологии с формированием необратимого повреждения миокарда связано с пролонгацией гиперкоагуля- 
ции, сосудистого спазма и других механизмов, начало и динамика которых определяется срывом системного регулирования. Сам факт критической гомеостатической неустойчивости в рамках уже имеющегося ОКС очевиден в связи с выходом традиционно контролируемых биологических параметров (ЧСС, АД, рН и др.) за допустимые границы. Вместе с тем начальный этап переходного процесса протекает скрыто, когда величины мониторируемых констант все еще близки к норме, а внутренние связи между функциональной активностью всего ансамбля органов и систем уже нарушены. Выявление факта этого явления и оценка степени его глубины может дать возможность найти соответствие между скрытыми проявлениями гомеостатической неустойчивости и вариантом течения ОКС БП ST в условиях неопределенности клинической симптоматики. Причем априорно ясно, что более выраженные изменения будут соответствовать не транзиторному, а необратимому патологическому процессу в миокарде. Поэтому принципиально описание функциональных расстройств не только качественного, но и количественного характера в рамках создания нового подхода к такой диагностике. Мерилом интенсивности патологического процесса могут выступать измеряемые характеристики переходного процесса в организации системного гомеостатического регулирования, который, как установлено в ранее проводимых исследованиях, является нелинейным $[4,6]$. В свою очередь, конкретным каналом получения информации об этих неявных сдвигах может быть оценка степени хаотичности такого высокоинформативного биологического сигнала как синусовый кардиоритм [5]. Целью проводимого исследования стало создание альтернативного подхода к объективизации функциональных расстройств, развивающихся на фоне клиники ОКСБП ST.

\section{МАТЕРИАЛЫ И МЕТОДЫ ИССЛЕДОВАНИЯ}

В исследование включили 60 больных ИБС с развитием ОКСБП ST (15 женщин и 45 мужчин), которые были госпитализированы по экстренным показаниям в блок интенсивной терапии (БИТ) отделения неотложной кардиологии. На вторые сутки пребывания в клинике диагноз исхода ОКС был окончательно верифицирован как нестабильная стенокардии III В класса по Ю. Браунвальду (HC) без повышения уровня тропонинов I и Т на фоне затянувшегося ангинозного приступа или не-Q ИМ, сопровождавшийся повышением уровня маркеров некроза миокарда на фоне типичной динамики ЭКГ, в связи с чем были сформированы 1 (НС) и 2 (ИМ) группы с равным числом боль- ных - по 30 человек в каждой. Средний возраст пациентов в этих выборках был 53,4 $\pm 5,6$ и 53,5 \pm 3,8 года соответственно. Длительность анамнеза ИБС составляла не более 5 лет, а симптоматика ухудшения состояния больных развилась в течение 1-2 суток. По данным признакам группы достоверных отличий не имели. Критериями исключения из исследования были инфаркт миокарда в анамнезе, стойкие нарушения синусового ритма, наличие сахарного диабета и выраженной декомпенсации сердечной недостаточности. Всем пациентам проводился лечебно-диагностический комплекс мероприятий, соответствующий национальным и европейским рекомендациям по диагностике и лечению ОКСБП ST. В рамках мониторирования гемодинамических параметров больных, находившихся в БИТ, проводился непрерывный анализ кардиоинтервалограммы и расчет относительной энтропии синусового кардиоритма в динамике. Для характеристики глубины функциональных расстройств выбрали 2-часовой период мониторирования в конце 2-х суток пребывания пациентов в БИТ, когда диагноз варианта обострения ИБС в рамках развития ОКСБП ST стал очевиден в связи с наличием или отсутствием в половине случаев реакции маркеров некроза миокарда - тропонинов и типичных феноменологических изменений ЭКГ. Структуру гомеокинеза «в глубину» выявляли, оценивая динамику его информационного показателя - энтропии, рассчитываемой по 200 последовательным интервалам RR c последующим постоянным сдвигом массивом на 1 дату. Даты энтропии кардиоритма составляли отсчеты ее фазовой траектории в многомерном пространстве состояний динамической системы. Поскольку расположение данных точек на этой детерминированно-хаотической траектории также оказалось случайно-подобным феноменом, анализ этой особенности позволил оценить сдвиги хаотичности организации управляющих многомодальных стимулов, которые модулировали варьирование длительностей RR-интервалов. Для удобства проведения количественного анализа хаотический процесс в каждом из индивидуальных случаев представили в виде аттрактора в n-мерном пространстве состояния системы. Дальнейшее описание сдвигов системного гомеокинеза реализовали путем статистического исследования цифровых параметров топологии аттракторов энтропии кардиоритма. После оценки нормальности распределения данных их статистическая обработка осуществлялась с помощью пакетов программ "Statistica", "Excel", "Statgragics". Результаты показаны в виде средних арифметических значений и их среднеквадратических отклонений $(\mathrm{M}+\mathrm{m})$. Межгрупповое сравнение проводили с помощью t-теста и критерия Стьюдента при $\mathrm{p}<0,05$. 


\section{РЕЗУЛЬТАТЫ ИССЛЕДОВАНИЯ И ИХ ОБСУЖДЕНИЕ}

Учитывая факт неравномерности расположения координат функционирования исследуемой системы на ее фазовой траектории, объективное представление о сдвигах хаотичности получили, оценивая графики плотности заполнения точками - отсчетами пространства существования (графики КСХ - коэффициента степени хаотичности), полученные при сканировании динамического процесса с пошагово изменяемой мерностью в диапазоне 20-220. Данная процедура позволила получить информацию об активности регуляторных процессов в частотном диапазоне порядка 10-120 сек. Интерпретация биологического значения выявленных при каждом шаге сканирования неравномерностей хаотического процесса, как одноапарметрической модели гомеокинеза, позволяет заключить, что сгущения на фазовой траектории и соответствующие им максимумы КСХ представляют собой участки, когда сдвиги параметра соответствуют текущим усложнениям структуры кардиоритма на фоне активации различных регуляторных механизмов одного конкретного иерархического уровня. Минимумы плотности соответствуют периодам текущих неустойчивых переходных состояний между соседними активированными режимами регуляции. Таким образом, топологические сгущения траектории - не что иное, как аттракторы 2-го порядка или субаттракторы. Это единицы структурирования основного притягивающего режима (то есть макроструктуры, соответствующей выбранной генеральной совокупности дат за 2-часовой период анализа). Накопление аналогичных данных за все последовательные циклы сканирования фазовой траектории до мерности, равной 220 , позволило охарактеризовать на заданную «Глубину» весь срез активности регуляторных механизмов, модулирующих синусовый ритм вплоть до процессов с 2-минутными периодами. В индивидуальных случаях в обеих группах обследованных лиц количество графиков с достоверно отличающейся формой кривых КСХ варьировало от 5-6 до 16-22. В 1 и 2 сравниваемых группах их число достоверно отличалось, составляя 10,42+1,44 против $15,41+3,32$ соответственно $(\mathrm{P}<0,05)$. Более детальная количественная статистическая оценка материала стала возможна после представления выявленных графиков КСХ в виде графа построения притягивающего режима для каждого из выявленных уровней. Анализ конкретных фазовых траекторий хаотических процессоров энтропии кардиоритма у всех обследованных показал, что они входят в области сгущений (субаттракторов) не последовательно, а путем сложных эволюций, включающих возвраты и блуждания между ними.
Координаты всех этих участков были найдены и идентифицированы с присвоением номеров, начиная с 1 и далее по признаку временной последовательности входа в них траектории. В результате были получены диаграммы - развертки появления и функционирования аттракторов 2-го порядка на каждом из уровней. Амплитуда столбиков графика с целью различения отдельных состояний соответствовала их порядковым номерам, а их длительность - времени нахождения траектории в данной фазовой области. Промежутки между столбиками диаграмм (фактически - изолиния) соответствовали переходу траектории от одного режима к другому, были равны реальному времени нахождения фазовой траектории вне субаттракторов. Далее разработали показатели для аналитического описания набора этих диаграмм в каждом из наблюдений в обеих группах: $\mathrm{M}$ - количество активированных режимов состояний (субаттракторов) на данном регуляторном уровне; Ms - число переключений этих состояний, то есть входов-выходов фазовой траектории в этих областях многомерного пространства; R - отношение числа активированных режимов и количества переключений; $\mathrm{Mb}$ - время, проведенное траекторией в основном режиме (наиболее устойчивом состоянии); $\mathrm{Mbs}$ - количество переключений основного субаттрактора; $\mathrm{Mr}$ - время, приходящееся на функционирование основного состояния относительно всех остальных активированных режимов без переходных процессов; Mt - время, проведенное траекторией в остальных (не основных) режимах; $\mathrm{Mz}$ - суммарная продолжительность переходных состояний, то есть нахождение траектории вне области притяжения субаттракторов.

В таблице 1 приведены средние значения статистических показателей топологии аттрактора энтропии кардиоритма у больных ОКСБП ST с исходом в НС (1-я группа) и 2-й группе с не-Q ИМ. Из нее следует, что в большинстве случаев сопоставлений величин параметров, полученных за данный конкретный 2-часовой период наблюдения, у больных с априорно разной выраженностью функциональных сдвигов гомеокинеза, связанных с обратимым (1-я группа) или необратимым (2-я группа) ишемическим поражением миокарда, имели место значимые различия. Характер выявленных изменений указывает на возникновение в случае более тяжелого исхода ОКС очевидных перестроек в организации системного регулирования с увеличением задействованных в текущий ответ большего числа иерархических уровней, к которым относятся механизмы, влияющие на структуру синусового кардиоритма и соответственно на величину его энтропии, отражающей многообразие этих модулирующих сигналов. Еще более существенным результатом 
Средние значения показателей топологии аттрактора кардиоритма в группах $(\mathrm{M} \pm \mathrm{m})$

\begin{tabular}{|c|c|c|}
\hline Показатели & $\begin{array}{c}1-я \text { группа (НС) } \\
\mathrm{n}=30\end{array}$ & $\begin{array}{c}2 \text {-я группа (ИМ) } \\
\mathrm{n}=30\end{array}$ \\
\hline $\mathrm{M}$ & $4,61+2,33^{*}$ & $6,95+3,32^{*}$ \\
\hline $\mathrm{Ms}$ & $49,82+28,41$ & $50,21+23,51$ \\
\hline $\mathrm{R}$ & $12,61+1,11^{*}$ & $8,04+0,71^{*}$ \\
\hline $\mathrm{Mb}$ & $0,240+0,032^{*}$ & $0,189+0,042^{*}$ \\
\hline $\mathrm{Mr}$ & $70,39+16,21$ & $68,11+13,45$ \\
\hline $\mathrm{Mb}$ & $28,44+10,24$ & $26,42+9,18$ \\
\hline $\mathrm{Mr}$ & $0,089+0,027^{*}$ & $0,137+0,031^{*}$ \\
\hline
\end{tabular}

Примечания: * - средние значения показателей, достоверно отличающиеся при сравнении групп $(\mathrm{P}>0,05)$.

является установление факта результативности использования для оценки выраженности функциональных расстройств параметров, описывающих информационно-энтропийную аттракторную модель гомеокинеза. В частности при появлении маркеров некроза миокарда достоверно нарастает эффект структурирования многомерного виртуального пространства состояний гомеокинеза, что подтверждается увеличением числа активированных дополнительных субаттракторов (параметр М). Вследствие этого фазовая траектория находится относительно меньше в основном режиме (показатель $\mathrm{Mb}$ ) и больше - во вновь возникающих локальных областях притяжения (Mt). Соответственно и время переходов между субаттракторами в реализациях 2 группы достоверно нарастает $(\mathrm{Mz})$ по сравнению с данными, выявленными в 1-й группе. Все эти вновь предлагаемые характеристики сдвигов системного гомеостатического регулирования относятся к анализу 2-го порядка, они отражают не сдвиги контролируемых параметров, а особенности процесса управления этими параметрами.

Таким образом, интегральный анализ функциональной нестабильности, возникающей при возникновении ОКС, позволяет получить объективное представление о ее выраженности, которая в свою очередь тесно взаимосвязана с сущностью соматических проявлений ИБС. В связи с тем, что данный подход основывается на характеристике сдвигов только одного параметра, мониторинг которого является стандартной процедурой, предполагается, что внедрение представленной выше технологии не приведет к дополни- тельными затратам и не создаст затруднений в накоплении информации, необходимой для количественной диагностики выраженности расстройств системного гомеостатического регулирования.

\section{ЛИТЕРАТУРА}

1. Бокерия О.Л., Биниашвили М.Б. Внезапная сердечная смерть и ишемическая болезнь сердца // Анналы аритмологии. - 2013. - № 2. - С. 69-79.

2. Бокерия Л.А., Бузиашвили Ю.И., Габетников Б.Г. Острый коронарный синдром. Возможности диагностики и лечения. - М. : Издательство НЦССХ им. А.Н. Бакулева РАМН, 2004. - 286 с.

3. Ватутин Т.Н., Смирнова А.С., Борт Д.В., Прокопенко Л.А., Узун Е.C. Обзор рекомендаций ESC 2015 года по ведению пациентов с острым коронарным синдромом без подъема сегмента ST. Часть I // Архивъ внутренней медицины. - 2016. № 2 (28). - C. 5-15.

4. Еськов В.М., Буров И.В., Филатова О.Е., Хадариов A.A. Основы биоинформационного анализа динамики микрохаотического поведения биосистем // Вестник новых медицинских технологий. 2012. - T. 19, № 1. - С. 15-18.

5. Сараев И.А., Довгаль В.М. Новые возможности диагностики на основе анализа нелинейных свойств гомеокинеза // Курск. науч.-практ. вестн. «Человек и его здоровье». - 2005. - № 2. С. 64-74.

6. Системный анализ и синтез в изучении явлений синергизма при управлении гомеостазом организма в условиях саногенеза и патогенеза / под ред. А.А. Хадарцева, В.М. Еськова. - Самара : Офорт, 2009. - Ч. 6. - 199 c. 